\title{
EGRESADOS DE ESCUELAS MEDIAS DE LA PROVINCIA DE NEUQUEN. SENTIDOS SOBRE EL MUNDO DEL TRABAJO, EL TRABAJO Y LA ESCUELA EN LA FORMACIÓN PARA EL TRABAJO.
}

\author{
Natalia Fernández (Universidad Nacional del Comahue)* \\ natinoe@hotmail.com
}

Recibido: 31/07/2012 Aceptado: 07/10/2012

\section{Resumen}

El presente trabajo se enmarca en un Proyecto de Investigación de la Universidad Nacional del Comahue denominado "Escuela Media y ciudadanía. Un estudio sobre prácticas pedagógicas en relación a la vida política y a la vida productiva en la Provincia de Neuquén", cuyo objetivo general del proyecto es estudiar prácticas pedagógicas de formación de ciudadanía que promueve la escuela media, en dos líneas de investigación: la vida productiva y la vida política. El presente artículo centra su mirada en la primera línea de investigación, entendiendo la formación para la vida productiva como las formas de vinculación que propone, realiza o genera la escuela con el mundo del trabajo. En este artículo indagamos los sentidos que sostienen los egresados referidos al mundo del trabajo, al trabajo y a la escuela en relación con el trabajo; de tres escuelas medias de la provincia de Neuquén.

Nuestra intención es recuperar los sentidos de estos/as jóvenes en tanto sujetos históricos y sociales, quienes construyen diversas significaciones en torno a su manera de ser y de estar en un contexto socio- histórico determinado.

\section{Palabras claves}

Egresados/as - Sentidos - Mundo del trabajo - Trabajo - Escuela en la formación para el trabajo.

\begin{abstract}
This work is in the framework of an investigation project called "Middle school and citizenship. A study about pedagogical practices in relation to political and productive life in Neuquén Province". Its general objective is to study pedagogical practices of
\end{abstract}

* Profesora en Ciencias de la Educación. Universidad Nacional del Comahue. 
citizenship formation that is proposed by middle school, in two central dimensions: productive life and political life.

The dimension in which this work is centered is in the productive life, understanding this theme, as the forms of entail that the school gives, realizes or generates with the world of work. In this article, we are interested in investigating the opinion of graduates about the world of work, work and school in relation with work; in three middle school of Neuquen Province. Our intention is to recover the senses of the young people as historical and social persons, who build different significances concerning their way to be in a social and historical framework.

\section{Key words}

Graduates - Senses - World of work - Work - School in the formation for the work.

\section{Introducción}

El presente trabajo se enmarca en un Proyecto de Investigación de la Universidad Nacional del Comahue denominado "Escuela Media y ciudadanía. Un estudio sobre prácticas pedagógicas en relación a la vida política y a la vida productiva en la Provincia de Neuquén" (1). Dicho proyecto se propone profundizar la mirada en torno a la constitución de ciudadanía en dos líneas de investigación: formación política y formación para la vida productiva.

Nuestro interés se centra en una de las líneas de investigación referida a la formación para la vida productiva, entendiéndola como las formas de vinculación que propone, realiza o genera la escuela con el mundo del trabajo. En este artículo indagamos los sentidos de los egresados referidos al mundo del trabajo, el trabajo y la escuela en la formación para el trabajo de tres escuelas medias de la provincia de Neuquén; a partir de entrevistas semi estructuradas realizadas en el año 2006 a dos egresados de cada institución.

Nuestra intención es recuperar los sentidos de estos jóvenes en tanto sujetos históricos y sociales quienes construyen diversas significaciones en torno a su manera de ser y de estar en un contexto socio- histórico determinado.

Para ello, este trabajo se estructura en cuatro apartados. En el primer apartado realizamos una breve caracterización de las tres instituciones educativas de nivel medio.

En el segundo apartado caracterizamos el modelo económico de la provincia de Neuquén y su mercado laboral en el período 2001-2006. Consideramos importante hacer esta aproximación para anclar los sentidos de los egresados en torno al mundo del trabajo, al trabajo y a la escuela en la formación para el trabajo. Sentidos que difieren en función de las significaciones que cada uno de los jóvenes tiene en su contexto de pertenencia. Situamos el período 20012006 ya que las entrevistas realizadas a los mismos datan del $2006 \mathrm{y}$, a su vez, dicho recorte temporal obedece a los datos estadísticos con los que contamos 
(Censo 2001 y datos provistos por el Ministerio de Seguridad y Trabajo de la Provincia de Neuquén -año 2005-).

A su vez, planteamos las condiciones 'subjetivas' de acceso al mundo del trabajo para los egresados/as, prestando especial atención al lugar del estudio en el ingreso al mismo.

En el tercer apartado realizamos una aproximación de las concepciones de los egresados respecto del trabajo y de los vínculos que plantean con sus propias proyecciones laborales.

Por último, nos centramos en la escuela en la formación para el trabajo, analizando los saberes que los egresados destacan en dicha formación y el lugar de la misma en la construcción de sus propias trayectorias.

\section{Consideraciones metodológicas}

Este trabajo se encuentra en el marco de una investigación de carácter cualitativo, para la cual, se realizaron entrevistas semi estructuradas a dos egresados/as -cuyos años de egreso van desde 2003 a 2005- de tres instituciones de nivel medio de la Provincia de Neuquén.

Las instituciones educativas de nivel medio analizadas son: una asentada en la región de los lagos (zona de San Martín y Junín de los Andes), otra en el centro de la provincia (zona Cutral Có- Plaza Huincul) y la última en la ciudad de Neuquén. Los criterios de selección de estas instituciones fueron: 1) las modalidades: las instituciones del interior son técnicas mientras que la de la capital es un bachiller; 2) el sector social que atienden: las del interior sectores medios y bajos y la de la capital concentra un sector socio económico medio- alto y 3 ) el tipo de gestión: las del interior son de gestión pública y la de la capital de gestión privada.

Respecto de los criterios de selección de los/as entrevistados/as fueron: alguien trabajando y alguien estudiando; un varón y una mujer.

La elección de egresados en este trabajo se realiza con el propósito de poder indagar el impacto que tiene la formación escolar en la construcción de sus trayectorias personales desde los sentidos que los jóvenes producen. Estos sentidos se entienden como una configuración dinámica en la que confluyen las significaciones previas construidas en el entorno social y familiar y, los elementos elaborados por ellos mismos en la propia experiencia escolar. Por lo tanto partimos de comprender que las significaciones que construyen los egresados provienen y son construidos desde una cierta narrativa. Nuestra intención es poder comprender dicha narrativa en torno al mundo del trabajo, al trabajo y a la formación escolar en torno al trabajo. 


\section{Los 'avatares' del mundo del trabajo en la Provincia de Neuquén: ¿cuál es el lugar de la juventud en esta esfera?}

Primeramente es importante realizar una breve caracterización del modelo económico de la Provincia del Neuquén para comprender el mercado laboral de la misma en el período 2001-2006. Nuestra intención es poder caracterizarlo en tanto nos interesa conocer los sentidos de los egresados en un contexto socio histórico determinado. Es decir, caracterizar las condiciones 'objetivas' del mercado laboral como las condiciones 'subjetivas' de los propios egresados/as en su vínculo con el mundo del trabajo y las proyecciones en el mismo.

Según Noya y Fernández (2004), el modelo de crecimiento económico de la provincia se puede caracterizar como exógeno de extracción y servicios asimilables a un capitalismo de enclave (2). Este tipo de economía extractiva (gas y petróleo) produce la dependencia en la renta petrolera y la escasa innovación e imposibilidad de desarrollar actividades industriales en otras esferas productivas. Es decir, más del $60 \%$ de la riqueza generada corresponde a la explotación del petróleo y gas, lo que provoca una alta dependencia de la ganancia que genera este recurso no renovable lo que, a su vez, produce pocos puestos de trabajo diversificado. Según Noya y Gerez (2010) este modelo económico provoca cierto riesgo a futuro de la sustentabilidad del modelo.

Frente a estas características es necesario profundizar las particularidades que asume el mercado laboral, siendo distinta la accesibilidad al trabajo según se trate de jóvenes o de adultos.

Si relacionamos la actividad económica con la población económicamente activa (PEA) (3), la rama más importante que concentra la población ocupada es el sector servicios con un $54 \%$, seguido del comercio con un $18 \%$ y, en el último escalón, se encuentra la explotación de minas y canteras. Sector que produce el mayor porcentaje de la riqueza provincial pero no cobra fuerza como generadora de empleo.

En el año 2005 (4), la población económicamente activa se concentraba en la franja etaria de los 30 a 49 años con más del $50 \%$ de ocupación, la franja de 25 a 29 años tibiamente esboza una ocupación del 15\%, lo mismo sucede con la franja de 20 a 24 años que alcanza sólo un $10 \%$. Con lo cual, la juventud de la provincia de Neuquén posee menores tasas de actividad y mayores tasas de desempleo. Como postula Noya y Fernández (2004) son los jóvenes una de las categorías sociales más afectadas por la desocupación. La composición de la población provincial hace prever que, año tras año, un número mayor de jóvenes irán incorporándose a la población activa, provocando -simultáneamente-que esos mismos jóvenes no logren encontrar trabajo a pesar de buscarlo.

Entonces podemos decir que el sector servicios concentra la masa laboral de la provincia. Es decir, la riqueza económica se centraliza en la actividad primaria de la explotación de recursos no renovables, pero el mercado laboral 
reúne la población ocupada en la rama de servicios, la actividad primaria (gas y petróleo) sólo cubre un tercio en la distribución de la población ocupada.

Estas características que asume el mercado laboral es una tendencia que se visualiza y se profundiza en estos últimos diez años. Según los datos proporcionados por la Dirección de estadística y censo (5), el sector servicios es el que aún concentra la mayor actividad laboral en todas las zonas que distingue a la provincia.

\subsection{Miradas de los/as egresados/as en torno al MUNDO DEL TRABAJO}

Consideramos importante analizar las condiciones 'subjetivas' en el ingreso al mercado laboral según la propia juventud. Juventud que no puede plantearse como un todo homogéneo sino que existen juventudes diversas en función de los condicionantes que afectan su manera de 'ser y estar en el mundo' y que los diferencian en función de condiciones económicas, educativas, laborales y relacionales que generan diferentes tipos "sociales" de jóvenes. (Salvia, 2008)

El mundo del trabajo se entiende como los modos de integración y ofertas que caracterizan el mercado laboral, en este caso, en la provincia de Neuquén. De este modo, resulta interesante pensar cómo es el proceso de integración de los jóvenes al mercado de trabajo, en particular, en el sector servicios que constituye la mayor parte de su oferta laboral.

En palabras de egresados/as:

Yo te digo, busqué, traté de ver. El tema que acá en Cutral Co no tenés muchas variantes para elegir el trabajo. O es comercio, o es limpiar en algún lugar, una casa o algo... Esas son tus variantes. $O$ sea, las posibilidades son limitadas. (Egresada, escuela técnica del interior de la Provincia, zona Cutral Co- Plaza Huincul. El resaltado es nuestro).

...Después entré a trabajar de albañil, y es un trabajo que a mí no me gusta, porque es esclavo ese trabajo, yo lo veo, que yo tengo un poco más de educación (...) porque yo dije, "estudié para estar haciendo esto" y eso te da bronca, porque en realidad yo tendría que estar trabajando en bromatología y las puertas no se me abrieron por un montón de cosas". (Egresado, escuela técnica del interior de la Provincia, zona de los lagos. El resaltado es nuestro)

En estas citas se puede registrar las limitaciones en la oferta laboral en las zonas del interior de la provincia, lo que reduce las posibilidades de trabajo. Por ejemplo, en la zona de Cutral Có- Plaza Huincul cobra importancia la producción hidrocarburífera, aunque prevalece el sector servicios como rama de actividad central; en el caso de la región de los lagos, que comprende San Martín de 
los Andes, Junín de los Andes y Villa La Angostura, se concentran actividades relacionadas al turismo pero, al igual que la zona anterior, el sector servicios es la rama que 'absorbe' el porcentaje de ocupados (6). La principal actividad económica de esta última zona está relacionada con el empleo público, si bien en los últimos años se ha notado un importante incremento en el desarrollo de la actividad turística, lo que ha aumentado notablemente la necesidad de mano de obra especializada en el área de Servicios.

Sin embargo, la última cita plantea la condición en la que accede el egresado al mercado de trabajo, quien tiene sus estudios secundarios finalizados pero su ingreso se limita al sector construcción cuando, en sus proyecciones, se visualiza en bromatología, área acorde al título técnico con el que egresa. Esta situación la plantea a partir de las pocas posibilidades que ha tenido en el ingreso a este sector. Estas ideas las podemos leer en función de la necesidad de tener influencias y contactos como mecanismos necesarios a la hora de acceder a un puesto de trabajo.

En palabras de egresados/as:

...Por ejemplo, acá en la destilería es difícil entrar... Aunque vos tengas el título es medio difícil, medio complicado porque es una empresa... es Repsol, es internacional, pero tenés que tener también... no sé, influyen varios factores, desde que tengas un conocido adentro y tengas fácil la entrada..." (Egresada, escuela técnica del interior, zona Plaza Huincul- Cutral Co. El resaltado es nuestro)

Es todo un manejo así de cosas... y las puertas para que uno ingrese si no tenés conocidos en la política no ingresás...por ahí. (...) Mirá yo terminé la secundaria y estoy trabajando en algo que no me gusta y que capaz que un pibe que está en la muni ahora no tiene la secundaría, o tiene la primaria hecha nomás y está en un puesto que, qué sé yo, se lo regalaron, porque tiene un tío, eso es un bajón. Yo creo que funciona así en todos lados. (Egresado, escuela técnica del interior, zona de los lagos. El resaltado es nuestro)

...y porque maestros faltan acá, pero de lo otro ya no (alimentos), creo yo no, o quizás no se tiene muy en cuenta al ser un pueblo tan chico no necesitan de lo que vos vas a estudiar, si te vas a un lugar más grande, sí, puede que consigas mas rápido trabajo, pudiendo ejercer algo de lo que estudiaste, pero no sé, en general, nunca se puede ejercer de lo que estudias casi nunca. (Egresada, escuela técnica del interior, zona de los lagos. El resaltado es nuestro)

En esta última cita se puede registrar que, al ser limitadas las ofertas en el mercado de trabajo, las necesidades se encuentran en otros campos laborales diferentes a los deseos y/o proyecciones en el estudio. En el caso de la región 
de los lagos parecería una 'necesidad concreta' la falta de maestros, tensionando la idea de 'pueblo chico' lo que provoca la necesidad de cubrir puestos de trabajo que quizás no responden a los estudios realizados.

Asimismo, en las expresiones de los egresados, se destaca la importancia de tener contactos como mecanismos de influencias necesarios en el mundo del trabajo. Dichos mecanismos pueden ser analizados, entre otros, por la relevancia que adquiere el partido político que gobierna con el mundo del trabajo de la provincia. Los 'contactos políticos' constituyen mecanismos 'filiatorios' necesarios en la integración al mundo del trabajo neuquino, donde el Estado provincial es la figura principal como fuente generadora de empleos, frente a una fuerza de trabajo creciente. "El papel del Estado provincial como activador de los mercados internos locales y protector de los sectores marginales, ha sido decisivo, pero, estuvo lejos de impulsar estrategias de reconversión de la estructura económica de la provincia". (Noya y Fernández, 2004: 192) Al existir un mecanismo de filiación estrecho entre trabajo y Estado, los contactos políticos son vías sugerentes para acceder a puestos de trabajos. Es necesario destacar que el Estado neuquino está fuertemente imbricado con el partido provincial (Movimiento Popular Neuquino); tal es así que se profundizan las prácticas clientelares que refuerzan y reconfiguran la identidad política. (Vaccarisi y Godoy, 2004).

A partir de los sentidos de estos egresados podemos pensar ¿cómo impactan en las propias proyecciones de estudio las 'condiciones de existencia' concretas que ofrece el mundo del trabajo en sus respectivas zonas? Pareciera una cuestión 'instalada' los contactos en el mundo del trabajo, entonces ¿cómo influye en las propias trayectorias escolares este sentido? Aspecto que nos detendremos profundizar en el punto 2.2.

Otra cuestión fundamental que plantean estos jóvenes es la falta de experiencia que poseen ante las demandas que genera el mundo del trabajo. Otros factores que juegan son la edad y los estudios secundarios como mecanismos excluyentes en el acceso a un puesto de trabajo.

En palabras de egresadas/os:

Eh... yo creo que... a veces miro los clasificados y se piden jóvenes de 18, 20 años con experiencia... y es como una contradicción importante lo que se está pidiendo, pero al margen de eso, no veo... (Egresada, escuela privada de la ciudad de Neuquén. El resaltado es nuestro)

Y, el principal problema para mi, es el estudio. El estudio, vos, por ejemplo ahora te exigen mucho terminar la secundaria para poder tener un trabajo, antes se exigía hasta la primaria nada más y ahora es bastante lo que te exigen. (Egresada, escuela técnica del interior, zona de Plaza Huincul- Cutral Có. El resaltado es nuestro) 
En estas expresiones se registra cierta vulnerabilidad en los jóvenes al acceder a un puesto laboral. Vulnerabilidad que se traduce en las problemáticas que ellos registran en el mundo del trabajo que se transforman en requisitos fundamentales a la hora de acceder a los mismos. El condicionante de la edad y de la experiencia laboral se puede visualizar como 'contradictorio' para quienes han terminado sus estudios secundarios. Es decir, no pueden acceder al mercado laboral por no tener, en algunos casos, experiencia laboral pero si la edad necesaria. Contradicciones que genera el propio mundo del trabajo; mundo que plantea el agotamiento del pleno empleo, donde la oferta de trabajo es reducida ante la demanda que existe.

Otro requisito es el estudio como aspecto imprescindible para obtener algún trabajo. Parecería que se constituye como mecanismo diferenciador en los puestos de trabajo. En este caso también están presentes las diferencias de los requisitos del 'pasado' con los de la actualidad. Hoy hay más exigencia, siendo necesario tener el título secundario para acceder a cualquier trabajo.

Según Salvia (2008), son numerosos los estudios que coinciden en señalar que los jóvenes constituyen un segmento poblacional especialmente afectado por los cambios ocurridos en el sistema productivo y la crisis de las instituciones públicas y sociales que tradicionalmente mediatizaban sus mecanismos de integración a la vida adulta (Salvia y Miranda, 1997; Jacinto, 2002; Salvia y Tuñón, 2003; Tuñón, 2005). Por ello, los lugares de ocupación laboral sólo se concentran en actividades mercantiles y de servicios, instituciones privadas que 'absorben' la población juvenil aunque su acceso a las condiciones de trabajo suelen caracterizarse como inestables y precarias.

Podemos decir que estos egresados destacan las influencias como mecanismos necesarios para conseguir empleo, en especial políticas, como también adquiere relevancia el estudio. Dos aspectos que quizás pueden 'leerse' como contradictorios ya que las influencias actúan como elementos 'arraigados' en el sistema, más allá de tener o no el título secundario y/o terciario en mano pero, a la vez, los egresados plantean la necesariedad de tener estudios. Aspecto que nos detendremos analizar en el punto siguiente.

\section{2 ¿Cuál es el lugar del estudio en el mundo del trabajo?}

Un aspecto que merece un análisis pormenorizado es el valor que los egresados destacan en el estudio como aspecto imprescindible y necesario en el mundo del trabajo.

En palabras de egresados/as:

Para mí la educación es todo, es una herramienta que mas hay que cuidar y que mas hay que valorar, que por ahí la gente no lo valora mucho, por ahí la gente no se da cuenta que el mayor nivelador de distintas clases sociales es la educación, es lo mas accesible y lo 
mejor para nivelar la clase social es lo que diferencia y por ahí no se valora tanto. (Egresado, escuela privada, ciudad de Neuquén. El resaltado es nuestro)

La desocupación, la pobreza y esto de que los chicos salen de la escuela sin un lugar donde ir a parar después y no tienen, por los bajos ingresos no pueden estudiar entonces terminan en el mundo del trabajo y no es que lo vea mal, pero me parece que si uno puede llenarse de más conocimientos lo veo mejor. Es un gran problema esto de no tener una posibilidad de estudio, de no darle tanta importancia nuestro país al estudio y que tenga que ser un sacrificio el estudiar. (Egresada, escuela privada, ciudad de Neuquén. El resaltado es nuestro)

En las voces de estos egresados la educación constituye una problemática central en el país, asumiendo que existen pocas posibilidades para los jóvenes de seguir estudiando. También es necesario destacar la institución educativa de la que egresan, en este caso pareciera haber similitudes en plantear la importancia de la educación y de su propia condición como 'privilegiados' al poder tener acceso al estudio, posibilidades que parecieran lograr pocos jóvenes. Esta situación provoca que la juventud acceda tempranamente al mundo laboral, mundo que, hemos visto, plantea mayores exigencias y requisitos a la hora de acceder a un puesto de trabajo como es, por ejemplo, el título secundario. Al aumentar los umbrales mínimos de escolaridad requeridos para puestos de trabajo de escasa calificación, el título de nivel secundario resulta indispensable para cualquier tipo de trabajo. Por otro lado, el tener niveles educativos más altos provoca mayores expectativas de mejor inserción laboral, que se contradicen con la realidad del mercado de trabajo, con lo cual los jóvenes tienen más tiempo de búsqueda y rotación que los adultos. (Alvarez, Bercovich, Herrero, Prugent, 2008)

A su vez, este problema que ubican en el estudio lo ven estos egresados como 'poco valorado' por las personas, al igual que se registra como mecanismo 'nivelador' de las clases sociales. Aquí es interesante pensar que esta 'poca valoración' está en las personas, es decir, es el propio sujeto quien tiene que 'encontrar' mecanismos para 'salir adelante'. Si queda en manos del sujeto, ¿qué lugar ocupa el Estado como garante y responsable de brindar educación? Estas ideas pueden estar en concordancia con la búsqueda de oportunidades que los propios individuos deben generar para su 'propio progreso'.

Respecto de los sentidos sobre la educación también están articulados con los consejos de sus familiares, quienes registran la importancia de los estudios para acceder a mejores puestos laborales.

...sí, mucho me recalcan (sus padres) que tengo que terminar de estudiar para recién poder conseguirme un trabajo, donde no me 
basureen, hay gente que se aprovecha de que uno tiene la primaria nada más y te basurean, mientras que ellos nada más tienen la secundaria, pero sí, me recalcan mucho el tema de poder tener un título en la mano para poder salir a trabajar dignamente que es una manera de decir, para poder trabajar bien, pero sí, eso es más que nada. (Egresada, escuela técnica del interior, zona de los lagos. El resaltado es nuestro)

... ella (la madre) sabe lo importante que es estudiar ¿viste? nunca me, como se llama, nunca me jodió para que estudie y nunca me presionó. Siempre fue, siempre me dejó esa libertad de poder decidir sobre mí, sobre lo que estoy haciendo y de qué manera lo hago. Y eso fue fundamental. (Egresado, escuela técnica del interior, zona de los lagos. El resaltado es nuestro)

En estas citas se registra la importancia de estudiar y de tener el título secundario para acceder a buenos puestos de trabajo. Estudios que también cobran sentido en tanto le permitirá al sujeto ser 'respetado' y trabajar 'dignamente', que podríamos pensarlo como aquellos trabajos que se reconocen los derechos del trabajador. A su vez se registra el estudio como mecanismo de ascenso y de movilidad social, siendo el puntapié para llegar a estos 'buenos trabajos'. Estas ideas pueden estar en sintonía con la impronta civilizatoria (7) moderna al concebir a la educación como vehículo fundamental para la futura inserción laboral. Se puede pensar en una relación lineal entre educación y trabajo, siendo el sujeto artífice del futuro y de su propio destino. Es decir, hay una apuesta en la educación como motor esencial en la búsqueda de trabajos.

En estos sentidos también es importante recuperar las propias proyecciones que tienen los egresados. Proyecciones vinculadas a seguir carreras terciarias.

Me gustaría en un futuro lejano, bastante por ahora, tener un jardín propio, ser la directora y bueno, manejar desde un lugar más tranquilo y no ser docente todo el tiempo porque creo que va a llegar una edad en que también me voy a cansar de tanto chico. Ojalá que no, que nunca me pase, pero también tener un lugar independiente, mío, propio, no ser siempre dependiente de alguien o de un estado o de un privado para trabajar. (Egresada, escuela privada de la ciudad de Neuquén)

A mi me encanta todo lo que es la parte de laboratorio. Me gusta mucho también lo que es el trato con las personas, a mí me encanta... de tener la posibilidad de vos relacionarte con otras personas. $Y$ tenés varias, no tan solo siguiendo carreras humanísticas, no, también estudiando enfermería tenés posibilidades. (Egresada, escuela técnica del interior, zona de Plaza Huincul- Cutral Có) 
...toda la vida quise trabajar de profesor de educación física, y sino de maestro. Si se me da la posibilidad de hacer algo más: joya. (Egresado, escuela técnica del interior, zona de los lagos)

En estas citas cobran sentido los gustos y los trabajos que implican estudios para poder realizarlo. Aquí se puede visualizar proyecciones a largo plazo cuya temporalidad es más diferida.

El estudio es el motor para poder concretar sus proyectos y que, podríamos decir, más allá de las características que han caracterizado en el mundo del trabajo, en especial los contactos e influencias, ellos apuestan al estudio.

Así, la educación seguiría constituyendo una "promesa" para los/as jóvenes, aún cuando perciban que no podrá ser cumplida con la sola terminación del nivel medio sino con la consecución de estudios superiores (Filmus, 2001).

Entonces se puede decir que el estudio es el motor fundamental para proyectar sus futuros profesionales, otro factor importante en sus proyecciones es el trabajo. Aspecto que nos detendremos a analizar en el siguiente apartado.

\section{Miradas de los y las egresados/as en torno al TRABAJO}

Consideramos que la escuela contribuye a la creación de imágenes y juicios que configuran no sólo la autoestima del estudiante sino que direcciona sus futuros trayectos.

Así también, el trabajo es otra esfera importante en la constitución de subjetividades. Neffa (2005) ofrece un aporte interesante para pensar al trabajo como una actividad amplia que tiene una utilidad social.

El trabajo es una actividad orientada hacia una finalidad, la producción de un bien o la prestación de un servicio que da lugar a una realidad objetiva exterior e independiente del sujeto y socialmente útil para la satisfacción de una necesidad. El trabajo involucra a todo el ser humano ya que también moviliza las dimensiones psíquicas y mentales (Neffa, 2005: 25).

Mediante el trabajo el sujeto construye su identidad social. Ahora bien, si pensamos la constitución del trabajo en la sociedad capitalista, éste se presenta 'resquebrajado'. Por ejemplo, hoy no existen habilidades ni experiencias que, una vez adquiridas, garanticen la obtención de un empleo y, en el caso de obtenerlo, este no resulta ser duradero. A su vez puede leerse este quiebre como 'crisis de representación' (Quiroga, 1990 en Figari, C y Dellatorre, 2004) en los sujetos. Esto alude a la fisura, las rupturas que se producen entre las expectativas de los sujetos y la realidad concreta. Frente a esta situación es necesario indagar ¿cómo se perciben los jóvenes en sus vínculos con el trabajo? Como señalamos anteriormente, el trabajo constituye una esfera importante en los procesos de construcción identitaria de los sujetos o, dicho de otro modo, 
el trabajo es un acto de producción del mundo y de transformación del sujeto. (Rabardel, 1995 en Spinosa, M, 2007).

Desde las voces de los y las egresados/as algunas de las miradas que emergen en torno al trabajo son las siguientes:

...trabajar? Dedicarle tiempo a algo que no sé, no te lo puedo describir así, pero sí, trabajar es algo, es dedicarle tiempo a algo que, qué bueno, que tenés que hacer sí o sí, o que necesitás hacer para poder sustentar necesidades que tengas (egresada, escuela técnica del Interior, zona de los lagos. El resaltado es nuestro)

...trabajar es buscarse la forma en la vida de conseguir las cosas que uno más anhela, es la forma de ser alguien, de encontrar dignidad, hay un montón de valores que nuestra sociedad crea como la dignidad, como la oportunidad, es la oportunidad de poder salir adelante, de tener/ uno dice que está mal ser materialista, hasta ahí nomás, no sé si está tan mal, es una forma de poder obtener todo lo material que uno necesita para ser feliz. (Egresado, escuela privada de la ciudad de Neuquén. El resaltado es nuestro)

Significa una independencia. Lo que significa independencia es tener mi propio dinero, aprender cosas nuevas, distintas que no se aprenden en una facultad, aprende de la vida, conectarse con otra gente, con otras cosas y también tener una independencia desde sentirse bien uno, mientras a vos te haga bien es importante que lo hagas. (Egresada, escuela privada de la ciudad de Neuquén. El resaltado es nuestro)

En estas expresiones se desprenden varios sentidos en torno al trabajo. Las últimas expresiones lo plantean como fuente de realización personal y desarrollo como sujeto social, centrando la importancia del trabajo en la dignificación del ser humano. No solo se busca la independencia económica sino que se espera que sea un medio para el bienestar, para 'ser feliz'. De esta manera el trabajo es visto como un aporte al crecimiento y libertad del sujeto. En este sentido se podría inferir que la temporalidad planteada es más diferida, es decir, trata de proyecciones futuras a largo plazo. En lo que respecta a la relación del sujeto con el trabajo, parecería ser más una relación de interioridad, en donde este contribuye a la constitución del ser, a la búsqueda de satisfacción personal y de bienestar (Martínez, Fernández, Ponzoni, 2007) (8). Asimismo es importante tener en cuenta que estas percepciones están atravesadas, entre otras, por la experiencia escolar. Experiencia que quizás esté relacionada con la propuesta que plantea la institución de residencia, cuya formación queda centrada en continuar estudios superiores. Así, el trabajo puede asociarse a proyecciones futuras en tanto impliquen estudios terciarios finalizados. 
Por otro lado, en la primera expresión se resalta al trabajo como un 'deber' que le compete al sujeto, asociado al sacrificio ante la necesidad de mantenerse. Este deber podría relacionarse con una visión instrumental del trabajo, en el sentido de realizar dicha actividad con el fin de satisfacer ciertas necesidades fundamentales del sujeto.

Desde otro plano también se plantea la 'dignidad' que se encuentra a partir del trabajo. En este caso se registra como un 'valor' social, estas ideas ¿estarán asociadas a un reconocimiento social? ¿Qué vínculos se pueden establecer entre trabajo y reconocimiento? Consideramos que existen vínculos estrechos entre ambos, pero creemos necesario seguir indagando en nuestra investigación.

De este modo podemos destacar dos significaciones en las concepciones sobre el trabajo: una referida a la subsistencia y otra representada por la realización y desarrollo personal y social.

Como podemos observar, hay indicios significativos para pensar que el trabajo constituye un factor primordial en la constitución identitaria de los egresados. Del mismo modo, sería interesante indagar la configuración de subjetividades en la formación para el trabajo que proponen las instituciones escolares. Aspecto que profundizaremos en el apartado siguiente desde las voces de los propios egresados/as.

\section{Miradas de los y las egresados/as en torno a la ESCUELA EN LA FORMACIÓN PARA EL TRABAJO}

La escuela en la Formación para el trabajo la entendemos como la formación escolar que brinda la institución respecto de las propuestas y vínculos que plantea con el trabajo. Los jóvenes plantean diversos sentidos que rescatan de la formación escolar para la proyección de sus trayectorias personales. Algunos destacan aportes que ofrece la escuela al desarrollo personal, subrayando la enseñanza de saberes, no sólo disciplinares, sino también aquellos que forman para la vida en sociedad. Hacemos alusión a las relaciones interpersonales y a la adquisición de ciertas responsabilidades que los egresados interiorizan como aspectos centrales en la formación para el trabajo.

En palabras de egresados/as:

E: que te parece importante aprender en la escuela, para desempeñarse en un trabajo? A: la responsabilidad, el aceptar normas, (...) aprender a, tampoco te digo que agachar la cabeza y que todo lo que te digan darlo por bueno, pero el saber convivir, la convivencia. (Egresada, escuela técnica del interior, zona Plaza HuinculCutral Có. El resaltado es nuestro).

...yo te digo este colegio me formó humanamente bien, es la mejor preparación que te pueden dar para un trabajo. Me enseñaron 
lo que es la dignidad, la responsabilidad, me enseñaron lo que vale el trabajo (...) porque el sentido de responsabilidad es muy importante para el resto de tu vida. (Egresado, escuela privada de la ciudad de Neuquén. El resaltado es nuestro)

...o sea, la responsabilidad ¿no? poder cumplir; eso es más bien personal, pero que la escuela también tiene un... como que es muy importante en la escuela, porque ya en los más chicos ya hacés un trabajo práctico por ahí nomás de a tres, por ahí éramos re chantas... Eso, después en la vida, es así como está la sociedad hoy ¿viste?". (Egresado, escuela técnica del interior, zona de los lagos)

Cabe aclarar que los sentidos en la formación escolar difieren dependiendo la institución en la cual los estudiantes egresan. Sentidos que se configuran en contextos determinados, tal como hemos analizado en apartados anteriores.

En estas citas podemos registrar que los egresados rescatan, en la preparación para un futuro trabajo, la importancia de la convivencia y la responsabilidad como aspectos centrales. Con relación a la responsabilidad, en la segunda expresión se registra ligada al ámbito interior de la persona (para sí) como forma de autorregulación, de internalización de la norma escolar y que tiene vínculos directos con la idea del uso de los derechos y obligaciones dentro de la escuela. Institución escolar que propone la autorregulación de la persona en pos de formar sujetos 'autónomos'. En este caso, el egresado plantea la responsabilidad no sólo para el ámbito del trabajo sino para la 'vida'.

En las demás expresiones, relacionadas al anterior, se plantea una responsabilidad como forma de cumplimiento, es decir con el 'otro', léase la escuela o en el ámbito de un trabajo. Estas dos formas están íntimamente relacionadas como una ida y vuelta. Del mismo modo podemos plantear que estas ideas pueden ser leídas en torno al 'disciplinamiento para el trabajo (9)' asociadas a comprender la 'gramática escolar' (Viñao, 2002) de la institución que se traduce en 'comportamientos' necesarios para la organización de su vida adulta y proyecciones en el mundo del trabajo.

Respecto de los conocimientos y saberes que destacan en la formación para el trabajo se plantean diversos aspectos.

En palabras de egresados/as:

Para el trabajo... en esto de relacionarme con otra gente, en exponerse. Yo aprendí mucho de eso, a aprender a valorarme por mí misma, yo siempre fui muy cabizbaja y siempre tenía la autoestima bastante baja (...) y bueno, a valorarse por lo que es uno y a ver que no es tan poca cosa y a valorarse y esto de exponerse también. (Egresada, escuela privada de la Ciudad de Neuquén. El resaltado es nuestro) 
...yo lo que aprendí mucho y lo que me gustó mucho fue el tema de alimentos, y realmente si vos necesitás un trabajo podés conseguir cualquiera, pero nos han formado más que nada en estos tres últimos años para eso, para poder trabajar en base a los alimentos. (Egresada, escuela técnica del interior, zona de los lagos)

Porque como yo te contaba que estaba estudiando el profesorado de geografía, por ahí es como que... mucho de lo que vos aprendés acá no lo podés aplicar pero (...) para mí es buena la base. (Egresada, escuela técnica del interior, zona Plaza Huincul- Cutral Có. El resaltado es nuestro)

De las citas expuestas, se puede registrar que los saberes están ligados tanto a la formación 'personal' como aquellos conocimientos ligados a la orientación de la escuela.

Respecto del primer aspecto constituyen saberes que se perciben como una herramienta fundamental en su futura inserción laboral. Herramientas que la escuela pareciera cumplir un papel central para el desarrollo de habilidades tales como la expresión y la exposición. Estas habilidades están presentes en la institución privada como objetivos explícitos en la formación del egresado/a.

En tanto los conocimientos ligados a la orientación constituyen un aporte esencial para definir (o no) las trayectorias laborales de los jóvenes.

Los jóvenes articulan una mirada de la escuela como instrumento (para el futuro) y como fin en sí misma (para aprender). Este último aspecto podría leerse como un reconocimiento de la escuela, teniendo en cuenta que estos estudiantes ya han finalizando el nivel medio.

No obstante, la evolución general de las tendencias no debe ocultar que las percepciones y prácticas de los actores están muy relacionadas con la diferente situación de los jóvenes frente a la desigualdad económica, social, política y geográfica.

Estas ideas conducen a pensar algunos interrogantes en torno a los espacios de inserción futura en el campo laboral y las prácticas escolares que habilitan/obstaculizan el proceso de formación del sujeto como ciudadano en un contexto socio histórico determinado.

\section{A modo de conclusión}

En este trabajo presentamos ciertos sentidos de los egresados referidos al trabajo, al mundo del trabajo y la escuela en dicha formación. Es interesante destacar que algunas de las proyecciones de los mismos pretenden 'superar' las condiciones de existencia que ofrece el mundo del trabajo. Así podemos pensar en los quiebres y rupturas que se visualizan entre las expectativas de los sujetos y de la realidad concreta; entre las tramas y vínculos de las condiciones objetivas con las subjetivas. Quiebres que ciertos egresados plantean 
ser conscientes de la situación social y económica inmersa. Es decir, varios caracterizan el mundo del trabajo a través de las limitaciones que encuentran en su acceso y de los contactos y/o influencias como mecanismos 'necesarios', en especial en las zonas del interior de la provincia. Sin embargo, ellos apuestan a seguir estudios superiores como herramienta y valor fundamental para acceder a buenos trabajos. Así, La educación sigue siendo una 'promesa', aún cuando perciban que quizás no podrá ser cumplida con la sola terminación del nivel medio. Por ello, la promesa se proyecta en la consecución de estudios superiores para visualizarse en un trabajo a partir de los gustos de cada uno de ellos. En este sentido cobra relevancia la impronta moderna en la configuración de destinos certeros a partir de entender a la educación como motor en las proyecciones laborales de dichos jóvenes.

Las profundas diferencias entre las situaciones y las expectativas de los egresados de distinto género, sector social, y contexto geográfico, nos ponen frente a la evidencia de la no linealidad de la relación educación -trabajo y del complejo entramado de las trayectorias individuales.

De esta manera, las miradas que los jóvenes poseen respecto al trabajo, mundo del trabajo y la formación escolar recibida, difieren dependiendo de los vínculos que establecen con la institución escolar de pertenencia, como de la ubicación geográfica, marcando diferentes significaciones. Por un lado encontramos miradas en torno al trabajo que enfatizan el desarrollo integral de la persona; por otro hay visiones que plantean el nivel instrumental del trabajo como medio para satisfacer las necesidades básicas de la persona.

Otro aspecto a destacar es la condición vulnerable de los jóvenes que se traduce en la contradicción del requisito de experiencia laboral a una población que aún no la tiene. Lo mismo sucede con la importancia de tener el título secundario, siendo condición necesaria, pero no suficiente, en el acceso a un buen trabajo. Aún más si tenemos presente la caracterización del mercado laboral de la provincia donde el sector servicios concentra la mayoría de la población ocupada. Existe una alta dependencia estatal en los puestos de trabajo, lo que profundiza la lógica clientelar en el acceso a los mismos. Es interesante advertir que se ha caracterizado el mercado laboral de la provincia del período 20012006 pero que existen tendencias significativas en la actualidad, según los datos que nos proporciona la dirección de estadística y censo. Tendencia que registra una alta concentración en el sector servicios (más del 50\%, dependiendo las zonas) y la mínima capacidad de generar empleo en las actividades primarias, en especial el sector hidrocarburífero. Ahora sería importante profundizar los sentidos que la juventud le otorga al mundo del trabajo hoy. Sentidos que, como vimos, están localizados en los contextos sociales de pertenencia.

Respecto de la formación escolar recibida en la formación para el trabajo, se destaca la importancia de la responsabilidad y la convivencia que han aprendido a lo largo de su trayectoria escolar. Aspectos centrales para pensar 
en el comportamiento 'esperado' en un trabajo. Estas ideas pueden estar en sintonía con la lógica del disciplinamiento en el trabajo. Este disciplinamiento también se puede analizar desde su configuración en las sociedades modernas. En esta dirección, Figari (2009) plantea:

La fuerza de la civilización normaliza y es hacedora de sujetos disciplinados, las fuerzas del capital gobiernan así un espacio/tiempo conformado técnica y socialmente para lograr ganancias y productividad. La disciplina industrial reclama la docilidad, que aporta civilidad (...) para recrear la armonía y la paz social. (2009:72).

Por ello cobra importancia, en las voces de algunos egresados, la norma escolar como espacio para hacer uso de las libertades y obligaciones en el marco institucional.

Estas ideas permiten interrogarnos sobre los sentidos que cada uno de los egresados construyen en torno a su manera de ser y de estar en un contexto socio histórico determinado; como también pensar en los saberes que la escuela genera en el proceso de formación de sujetos en tanto ciudadanos productores, que define-orienta-produce diversos destinos laborales en los sujetos.

\section{Notas Bibliográficas}

(1) Dicho proyecto tiene su antecedente más inmediato en un proyecto denominado "Desigualdad y procesos escolares de construcción de identidades de género en seis escuelas medias de la provincia de Neuquén. Articulaciones entre formación política, formación para el mundo de trabajo y prácticas de lectura y escritura en la producción de ciudadanía”. En este proyecto -que finalizó en el 2009- el objetivo se orientó a caracterizar las formas escolares de producción y reproducción de las desigualdades de género en su particular articulación con las desigualdades de clase, etnia, edad, nacionalidad, mediante un estudio en seis establecimientos de nivel medio - de distintas modalidades, gestiones y niveles socioeconómicos de la población que asiste- de la provincia de Neuquén. En este marco se realizó el trabajo de campo que continúa con su línea de análisis en el proyecto vigente.

(2) Extracción del recurso natural hasta el agotamiento, con consecuencias en la estructura del trabajo y en la distribución del ingreso. (Noya y Fernández, 2004)

(3) Según el censo 2001.

(4) Estos datos fueron proporcionados por la Subsecretaría de empleo y capacitación. Ministerio de seguridad y trabajo de la Provincia del Neuquén.

(5) $3^{\circ}$ trimestre de 2010

(6) Según la caracterización del mercado laboral en el apartado 2.1. Asimismo es importante advertir que los datos se mantienen en la actualidad ( $3^{\circ}$ trimestre de 2010$)$ según la Dirección de estadísticas y censos de la Provincia de Neuquén.

(7) El orden capitalista configura un sistema ideológico que se impone como patrón civilizatorio que genera un orden discursivo y legitimador nutrido por la ideología liberal y fortalecido por las instituciones 'disciplinarias' (Foucault, 1995) que construyen un tipo de sujeto. (Figari, 2005) 
(8) Estos análisis se han desarrollado en una versión anterior presentada en las I Jornadas de Investigación en Educación "Sujetos, prácticas y alternativas". Mar del Plata. Mayo de 2007; junto con las investigadoras Martinez Silvia y Ponzoni Elena.

(9) Esta idea está ampliamente desarrollada en Martinez, Fernández, Ganem (2009).

\section{Referencias Bibliográficas}

- $\quad$ Alvarez, M; Bercovich,G; Herrero, A; Prugent, B (2008) Educación y trabajo. El caso del interior de la Provincia de Neuquén. Dirección provincial de censo y estadística de la Provincia de Neuquén.

- $\quad$ Figari, C; Dellatorre, G (2004)“Universidad y educación en las representaciones de los jóvenes que estudian educación. Viejos y nuevos sentidos del mandato moderno. Revista Argentina de Sociología Año 2 N³. (40-55)

- $\quad$ Figari, C (2009) Prácticas corporativas empresariales y disciplinamiento social/cultural: desnaturalización y crítica a la pedagogía empresaria. En Figari, C; Alves, G (Orgs) La precarización del trabajo en América Latina. Perspectivas del capitalismo global. (6994) Brasil, Praxis.

- Martínez, S; Fernández, N y Ponzoni, E (2007) Miradas/sentidos de los alumnos y alumnas de dos escuelas técnicas en la formación para el trabajo. I Jornadas de Investigación en Educación "Sujetos, prácticas y alternativas". Mar del Plata. Mayo de 2007.

- $\quad$ Martínez, S; Fernández, N y Ganem, M (2009) Escuela Media, contextos y discursos sobre el trabajo en la Provincia de Neuquén. Revista Ciencias de la Educación 5 (4) (155-182)

- $\quad$ Neffa, Julio (Coord.) (2005) Actividad, empleo y desempleo: conceptos y definiciones. Buenos Aires: Miño y Dávila Editores.

- $\quad$ Noya, N y Fernández, N (2004) Modelo económico de la Provincia de Neuquén. Funcionalidad de las políticas activas del gobierno provincial. En Rafart, G; Quintar, J; Vela, F (2004) 20 años de democracia en Neuquén y Río Negro. (191-208) Neuquén: Educo.

- Noya, N y Gerez, L (2010) Desarrollo de la Ciudad de Neuquén. Diagnóstico y perspectivas futuras de acuerdo con la gestión del gobierno local. IV Jornadas nacionales de investigadores de las economías regionales. Universidad Nacional de Entre Ríos.

- Salvia, A (comp.) (2008) Jóvenes Promesas. Trabajo, educación y exclusión social de los jóvenes pobres en la Argentina. Buenos Aires: Miño y Dávila.

- Spinosa, M (2007) El análisis y reconocimiento del saber en el trabajo. V Congreso Latinoamericano de Sociología del Trabajo. Abril, Montevideo, Uruguay.

- Vaccarisi, A y Godoy, A (2004) Estado Neuquino: entre la hegemonía del partido provincial y las oposiciones. En Rafart, G; Quintar, J; Vela, F (2004) 20 años de democracia en Neuquén y Río Negro. (109-126) Neuquén: Educo.

- Viñao F (2002) Sistemas educativos, culturas escolares y reformas: continuidades y cambios. Madrid. Morata (versión digital) 